Archives

3| 1989

Varia

\title{
Une banque de données en développement sur la mécanisation de la France
}

\section{Serge Benoit, Gérard Emptoz et Claudine Fontanon}

\section{(2) OpenEdition}

Journals

Édition électronique

URL : http://journals.openedition.org/ccrh/2919

DOI : $10.4000 /$ ccrh.2919

ISSN : $1760-7906$

Éditeur

Centre de recherches historiques - EHESS

Édition imprimée

Date de publication : 15 avril 1989

ISSN : 0990-9141

Référence électronique

Serge Benoit, Gérard Emptoz et Claudine Fontanon, « Une banque de données en développement sur la mécanisation de la France ", Les Cahiers du Centre de Recherches Historiques [En ligne], 3 | 1989, mis en ligne le 13 avril 2009, consulté le 20 avril 2019. URL : http://journals.openedition.org/ccrh/2919 ; DOI : $10.4000 /$ ccrh.2919

Ce document a été généré automatiquement le 20 avril 2019

Article L.111-1 du Code de la propriété intellectuelle. 


\title{
Une banque de données en développement sur la mécanisation de la France
}

\author{
Serge Benoit, Gérard Emptoz et Claudine Fontanon
}

1 Dans le processus d'ensemble de la première industrialisation, la mécanisation occupe une place centrale: extension dans la sphère de la production, sur une échelle sans précédent et avec une tendance à la généralisation, du machinisme comme remplacement du travail manuel; utilisation sans cesse croissante, à cette fin, d'énergie mécanique inanimée accompagnée d'un renouvellement des sources de celle-ci; application au secteur des transports de la motorisation auparavant limitée aux activités manufacturières de type proto-industriel, avec l'avènement de moteurs installables sur des véhicules.

2 Cet essor de la mécanisation à travers ces diverses manifestations a été rendu possible par l'apparition, contemporaine des débuts mêmes de la révolution industrielle, d'un secteur industriel à part entière, totalement nouveau: la construction mécanique. Industrie spécifiquement issue de l'industrialisation, celle-ci est aussi l'une des conditions absolues de son développement. Elle forme, en outre, l'un des rameaux de la métallurgie différenciée moderne, dans la mesure où ses réalisations n'ont été permises que par les percées technologiques effectuées par la sidérurgie en Grande Bretagne au cours du XVIII siècle : emploi du combustible minéral abaissant radicalement le coût du métal ; mise au point de nouveaux instruments permettant de diversifier sans cesse les fabrications: fourneau à réverbère à la Wilkinson pour le travail de la fonte de fer en seconde fusion, permettant de multiplier les objets de moulage, de plus en plus complexes de surcroit ; laminoir pour l'élaboration, à un coût sans cesse abaissé, de produits plats et longs, toujours plus diversifiés.

3 Inséparable de la révolution des matériaux, sans laquelle n'auraient pu être mis au point ni les nouveaux moteurs primaires actionnant les manufactures, ni ceux propulsant les véhicules terrestres et marins, non plus que les machines aux capacités de production indéfiniment extensibles de la nouvelle organisation usinière, la construction mécanique 
se situe au coeur même du système technique et économique apparu à la fin du XVIII ${ }^{\mathrm{e}}$ siècle et devenu dominant au milieu du suivant. Elle en apparaît comme l'un des piliers, en même temps que comme l'un des moteurs, jouant un rôle d'entraînement vis-à-vis de la croissance générale de l'économie aussi décisif que les charbonnages, la sidérurgie et l'industrie cotonnière.

4 Aux origines de l'industrialisation, la construction mécanique représente l'un des éléments constitutifs du corps des «techniques anglaises ». Aussi bien les initiateurs de celle-ci en France sous cette forme furent-ils pour la plupart des techniciens britanniques, dont on regrettera que le rôle capital, magistralement mis en lumière, voici près de 70 ans déjà, dans l'oeuvre pionnière de Charles Ballot ${ }^{1}$, n'ait pas été depuis lors davantage approfondi, en dehors de travaux comme la contribution, de premier ordre celle-là, de Serge Chassagne ${ }^{2}$ sur la naissance de l'industrie cotonnière.

Il n'en demeure pas moins que, passé cette phase initiale relevant typiquement de l'importation et du transfert de technologie, la construction mécanique s'est rapidement naturalisée en France, au point d'y donner lieu à une véritable école nationale ayant fait preuve d'originalité dans plus d'un domaine. C'est cette floraison créatrice que célèbre le Musée centennal de la Mécanique française, publié par Eude à l'occasion de l'Exposition universelle de Paris-1900. La présence et le rayonnement international de ce secteur de l'industrie française au siècle dernier n'en contrastent que davantage avec sa situation actuelle, où, à côté de certaines réussites remarquables dans le domaine des turbomachines de toute nature (hydrauliques, à vapeur aussi bien qu'à gaz), il apparaît, en particulier dans celui de la machine-outils, comme l'un des plus sinistrés de la dernière période, en passe, à certains égards, d'être rayé de la carte.

6 Les multiples interrogations que suscite une telle évolution en longue durée ne font que souligner d'autant plus l'urgence et la nécessité d'en promouvoir l'analyse historique. Celle-ci reste, en effet, encore très largement à entreprendre, en dehors du monde des mécaniciens de la capitale qui ont déjà donné lieu à des recherches importantes et variées dans leur inspiration : en particulier celles poursuivies, en termes de filières techniques, au Centre de Documentation d'Histoire des Techniques ${ }^{3}$ à l'initiative de Maurice Daumas et Jacques Payen ; celle de James Edmonson, sous l'angle de la formation intellectuelle et professionnelle des constructeurs ; ainsi que celle, en cours, de Jacques Retel, axée sur la notion de réseau industriel au sein d'une grande agglomération urbaine. La mécanique française de cette période est, en effet, loin de se ramener à ce milieu parisien, si fécond et si important soit-il, et doit être envisagée dans ses implantations provinciales, de plus en plus dispersées à mesure que l'on s'éloigne de la phase initiale de son développement, confirmant par là-même sa naturalisation en profondeur à travers le tissu productif.

\section{Un observatoire privilégie : la construction des moteurs hydrauliques au siècle dernier}

$7 \mathrm{Au}$ milieu de ce secteur qui a connu une véritable efflorescence dans de multiples directions, une attention toute particulière doit être accordée au domaine des moteurs primaires, et notamment aux moteurs fixes employés dans l'industrie.

8 Si cette dernière notion renvoie assurément, pour cette période, au développement de la machine à vapeur alternative, il reste que l'usage de cette dernière ne se généralisa dans les manufactures françaises qu'à une époque tardive, relativement à d'autres pays tels 
que l'Angleterre ou la Belgique. Jusqu'aux années 1850-1860, en effet, correspondant à un stade déjà avancé de l'industrialisation, ce fut l'énergie hydraulique qui demeura la principale source de force motrice, y compris dans des secteurs d'entraînement aussi essentiels que la sidérurgie primaire ou le textile mécanisé.

Ce rôle persistant de l'hydraulique comme base énergétique prépondérante de la croissance industrielle française ne peut plus être considéré aujourd'hui ni comme un symptôme d'archaïsme, ni comme un cas isolé. Les nombreux travaux menés OutreAtlantique depuis une vingtaine d'années ont, au premier chef, achevé de démontrer la place décisive, comme la modernité de cette source d'énergie dans l'industrialisation américaine jusqu'à la Guerre Civile, cependant que dans bien d'autres pays ou régions du Vieux-Continent, de l'Europe méridionale (Italie du Nord, Catalogne) aussi bien que nordique (pays scandinaves, Finlande) ou centrale (Suisse), les ressources des cours d'eau n'ont pas cessé de tenir une place primordiale, avant de trouver un rôle encore plus étendu avec la production électrique.

C'est en fonction de ce renversement des perspectives sur les bases énergétiques de la première industrialisation qu'il faut envisager la facture des moteurs hydrauliques en France au $\mathrm{XIX}^{\mathrm{e}}$ siècle comme domaine privilégié pour l'analyse de la voie nationale de la croissance et du progrès technique.

11 Au-delà de cette place tenue dans le système productif, l'utilisation de l'énergie hydraulique en France au siècle dernier a donné lieu, sur le plan proprement technologique, à des développements originaux majeurs de portée internationale. Bertrand Gille comme Maurice Daumas avaient, chacun, contribué à mettre en lumière le rôle pionnier joué par la France, durant la première moitié du XIX ${ }^{e}$ siècle, pour la mise au point des premiers moteurs hydrauliques modernes, et notamment de l'innovation majeure que constituait la turbine, dès les années 1820-1830. Il s'est agi, de surcroît, d'un domaine central, dans l'historiographie française aussi bien qu'étrangère, pour l'étude des relations entre science et technique à l'aube de la civilisation industrielle, puisqu'aussi bien de nombreux auteurs ont soulevé le problème de l'influence exercée, dans ces avancées techniques françaises, de la mécanique rationnelle des fluides à laquelle l'école physico-mathématique du XviII ${ }^{\mathrm{e}}$ siècle avait apporté une contribution de premier ordre.

12 Ainsi s'est constituée, autour du thème des moteurs hydrauliques, toute une problématique d'ensemble intégrant les rapports entre invention et innovation, théorie scientifique et pratique technique, technologie et développement industriel. C'est cette pluralité d'aspects qu'une série de recherches collectives, gravitant autour du Centre de Recherches Historiques, se sont attachées, depuis une dizaine d'années, à saisir dans leur diversité en même temps que dans leurs imbrications. Il s'est agi de trois programmes placés les uns et les autres sous la responsabilité scientifique de Louis Bergeron, qui ont été menés à bien depuis 1981 :

- Le programme d'inventaire expérimental des roues hydrauliques, réalisé en 1981-1982, dans le cadre d'un contrat passé avec la MIDIST, doublé d'un autre contrat avec la Mission du Patrimoine Ethnologique au Ministère de la Culture ${ }^{4}$;

- la recherche effectuée en 1983-1984 au titre du Programme "Science, Technologie et Société » du C.N.R.S., sur «les relations entre conception et réalisation, tradition et innovation dans le domaine des moteurs hydrauliques $»^{5}$; 
- en troisième lieu, l'action conduite dans le cadre de l'A.T.P. du C.N.R.S. «Histoire industrielle de la France » en 1985-1987, sur les inventeurs, constructeurs et ingénieurs, portant sur le même secteur et la même période ${ }^{6}$.

13 Pour pouvoir continuer d'approfondir cet axe de recherches, dont la richesse est encore loin d'être épuisée, il est apparu indispensable, à l'issue de ces trois actions, de se doter d'un instrument approprié, sous la forme d'une banque de données informatisée, pour gérer et exploiter systématiquement la masse d'informations accumulée en l'espace d'une décennie.

\section{Un outil au service d'une équipe de recherche : la banque de données sur la mécanisation de la France à l'ère de l'industrialisation}

\section{Un champ progressivement étendu à l'ensemble du secteur de la mécanique}

L'étude en profondeur de la facture des moteurs hydrauliques en France au siècle dernier a fait apparaître que celle-ci ne constituait pas à cette époque une activité autonome, comme elle $\mathrm{a}$, au contraire, tendu à le devenir à partir de la fin de la période et comme c'est le cas aujourd'hui. Cet état de choses n'avait, du reste, rien de propre à ce domaine technique ; il en allait de même des autres types de productions relevant du secteur de la construction mécanique.

Dans le cas le plus fréquent, un constructeur produisait la totalité des équipements nécessaires à une ou plusieurs branches industrielles spécifiques de manière à pouvoir fournir ce que l'on appellerait de nos jours des usines clés en main, depuis les machines motrices (à vapeur aussi bien qu'hydrauliques) jusqu'aux appareils de fabrication les plus spécialisés (laminoirs, presses, cisailles, marteaux-pilons dans le cas de la sidérurgie, butoirs, chaines à godets, sasseurs, dans celui de la meunerie, etc.), en passant par les indispensables mécanismes de transmission du mouvement (engrenages, arbres, poulies et courroies, etc.), désignés sous le terme générique de millwork dans la littérature technique anglo-américaine. Ce fut ainsi le cas, pour ne citer que quelques-uns des plus notables d'entre eux, des firmes Schneider ou encore Crozet-Fourneyron pour l'industrie lourde, Fontaine pour la meunerie, De Canson pour la papeterie, Koechlin (l'une des principales composantes historiques de l'actuelle Alsthom) pour le textile cotonnier, et il ne manque pas d'exemples pour montrer que cette situation se retrouve à l'étranger, avec des noms aussi majeurs actuellement dans le domaine des turbines hydrauliques que celui de la firme helvétique Escher-Wyss (aujourd'hui intégrée au Groupe Sulzer) ou de son homologue allemande J.-M. Voith, qui produisaient aussi à l'époque des machines textiles dans le cas de la première, et des équipements pour les râperies et papeteries dans celui de la seconde.

Il est donc possible maintenant d'étendre le constat ainsi dressé par Serge Chassagne à propos des matériels destinés à l'industrie cotonnière, en affirmant qu'au siècle dernier les constructeurs mécaniciens étaient, en règle générale, polyvalents sans être nécessairement des généralistes. Dans ces conditions, il n'est guère de constructeurs mécaniciens établis en France-car la remarque s'applique tout aussi bien aux techniciens d'origine britannique venus s'installer de ce côté-ci de la Manche-, qui 
n'aient, à des degrés divers, à un moment ou à un autre, produit des moteurs hydrauliques. A étudier les fabricants de ce type de machines motrices, c'est en réalité l'ensemble de la mécanique française que l'on tend à atteindre et à cerner dans ses contours et ses structures essentielles.

\section{Une approche axée sur les acteurs de l'innovation} moteurs hydrauliques ouvrait des fenêtres sur l'ensemble du secteur de la mécanique, le courant de recherches rassemblé autour du C.R.H. a délibérément choisi de privilégier une approche axée sur les différents acteurs, individuels ou collectifs, de l'innovation, depuis que l'étude intermédiaire réalisée en 1983-1984 a achevé de mettre en lumière l'exceptionnelle floraison dont la France avait été le théâtre durant la majeure partie du siècle dernier dans ce champ particulier de la création technique.

En plus des constructeurs mécaniciens proprement dits, occupant une position centrale dans ce processus, il est apparu indispensable de prendre en compte, dans toute leur diversité, les autres intervenants en amont aussi bien qu'en aval de ceux-ci, dans leur rôle de concepteurs, d'utilisateurs ou encore d'intermédiaires, notamment dans le passage du stade de l'invention à l'innovation, désignés ici sous le terme générique de médiateurs. Il s'est donc principalement agi, dans le cas présent, des types suivants d'acteurs :

- les ingénieurs civils, qui préfigurèrent au XIX ${ }^{e}$ siècle nos bureaux d'études contemporains, prenant part à la conception mais non à la réalisation, et faisant exécuter par des constructeurs mécaniciens professionnels les projets établis par leurs soins (ainsi Benoît Fourneyron faisant réaliser ses turbines, avant 1850, par des firmes françaises telles que les maisons Koechlin ou De Dietrich ; ou étrangères comme celle de William Fairbairn);

- les cabinets d'ingénieurs-conseils en propriété industrielle - dont le cabinet Armengaud Aîné, en même temps éditeur de premier plan en matière de publications techniques et professionnelles, constitue l'archétype -, jouant un véritable rôle de plaque tournante pour le dépôt des brevets d'invention et leur exploitation à travers les cessions de droits et les licences, de bourse de l'innovation à bien des égards ;

- les membres des instances scientifiques et techniques officielles ou officieuses, ayant exercé, dans le cas de la France, une fonction majeure d'incitation au progrès technique et d'évaluation des différentes innovations en présence: Académie des Sciences, Société d'Encouragement pour l'Industrie nationale, Jurys des grandes expositions nationales et universelles, membres de ces formations spécifiquement françaises que sont les appareils techniques d'Etat, civils (Corps des Mines ou des Ponts et Chaussées) ou militaires (Artillerie, Génie, Marine) ;

- les institutions de formation professionnelle, et notamment les grandes écoles d'ingénieurs, comme lieu de transmission des connaissances, dans leurs rapports avec le mouvement des idées scientifiques et des pratiques techniques;

- à l'autre extrémité de la chaîne, enfin, les utilisateurs, ici les manufacturiers employant l'énergie hydraulique, comme destinataires finaux des innovations, mais aussi comme auteurs de la demande de progrès technique.

Il est apparu que, pour cerner ces acteurs dans le cadre d'une grille d'analyse commune, la démarche la plus appropriée devait être ici aussi de type prosoprographique, à l'instar de celle qui fait aujourd'hui toutes ses preuves à l'égard de catégories sociales telles que les grands notables ou les chefs d'entreprises. A l'intérieur du vaste champ concerné par 
la mécanique, la participation à l'innovation de ces différents acteurs les assimile, en effet, à une élite, en sorte qu'il parait donc tout à la fois légitime et souhaitable de les aborder au moyen de méthodes similaires. Il s'agit bien, à chaque fois, de prendre en compte leurs origines et leurs alliances familiales, leurs formations, leurs carrières (professionnelles, au premier chef, mais aussi, le cas échéant, associatives et politiques), leurs productions (celles-ci pouvant consister, dans le cas présent, aussi bien en brevets d'invention ou en machines effectivement réalisées qu'en publications ou en études opérationnelles), ainsi que les éléments de mesure de leur réussite (revenus, patrimoines, honneurs publics).

\section{La mise en oeuvre des objectifs assignés à la banque de données MEDOC : aspects techniques} données sur la mécanisation de la France vise, sur le plan de la gestion documentaire, à permettre l'exploitation d'une pluralité de fichiers de contenus diversifiés, réalisés par des chercheurs de différentes disciplines, selon des approches variées. L'outil a donc une vocation fédérative, sur la base d'un dénominateur commun, par rapport aux fichiers existants et aussi vis-à-vis de ceux qui seront créés ultérieurement.

L'architecture de cette banque de données s'ordonne actuellement autour de quatre types de notices normalisées :

- $1^{\circ}$ le fichier des inventeurs, constitué pour l'essentiel à partir du fonds, dépouillé exhaustivement, des brevets conservés à l'Institut national de la Propriété industrielle se rapportant aux moteurs hydrauliques, pour la période 1791-1918, qui concerne un corpus de quelque 770 brevets retenus comme pertinents, à l'issue d'un tri sévère ayant porté sur plus de 1500 brevets identifiés d'après leur titre ;

- $2^{\circ}$ le fichier des sources biographiques et bibliographiques relatives aux acteurs de l'innovation, provenant de la littérature professionnelle et technique de l'époque, incluant également de manière systématique les rapports de toutes les expositions nationales et universelles, ainsi, de manière plus aléatoire et occasionnelle, que des références et documents d'archives publiques et privées (près de 500 fiches actuellement);

- $3^{\circ}$ le fichier des dossiers individuels de synthèse réalisés à partir des informations précédentes à propos d'un nombre restreint de personnages (cf. infra) faisant l'objet de monographies;

- $4^{\circ}$ le fichier des entrepreneurs et des entreprises, construit principalement à partir du fichier du C.D.H.T. sur la mécanique parisienne, d'ores et déjà complété par un certain nombre de données sur la province et prenant également en compte les ingénieurs dits civils tels qu'ils ont été définis plus haut.

Il s'agit donc de notices articulées autour des individualités, avec des rubriques communes et des descripteurs identiques :

- données professionnelles : ex. Mécanicien;

- secteur d'activité : ex. Construction mécanique, Machine-outil, etc.;

- données économiques : Chiffre d'affaires, Effectifs, etc. ;

- données techniques : ex. type de machine (Turbine axiale, Roue de côté à augets, etc.) ;

- données géographiques : adresse, commune, département, pays ;

- données professionnelles : ex. Mécanicien; 
- données iconographiques : portraits, plans, dessins et représentations des usines ou de leurs productions. entreprise, ingénieurs civils, dirigeants de cabinets-conseils, ingénieurs militaires, professeurs de grandes écoles, industriels utilisateurs de l'énergie hydraulique devenus innovateurs. 
31 ans dire qu'un tel produit, de caractère probatoire, n'est qu'une voie parmi d'autres pour mettre de l'ordre et dégager un sens au milieu du foisonnement créateur auquel a donné lieu le secteur de la mécanique à l'ère de l'industrialisation. D'autres formes d'exploitation de cette banque de données, cherchant à atteindre une plus grande exhaustivité et non limitées au seul dénominateur commun de l'hydraulique, pourront voir le jour par la suite, en fonction de la poursuite de la mise en oeuvre des fichiers existants et d'autres dont la création est envisagée actuellement ${ }^{7}$. Ce projet doit être envisagé comme une étape dans une recherche installée aujourd'hui dans la longue durée, dont les antécédents ont déjà été publiés ou sont en voie de l'être très prochainement.

\section{NOTES}

1. L'introduction du machinisme dans l'industrie française, Paris, 1923.

2. Naissance de l'industrie cotonnière, 1760-1840. Trois générations d'entrepreneurs. Thèse de doctorat d'État, E.H.E.S.S., 1986, Lille, Atelier de reproduction des thèses.

3. Centre commun du C.N.A.M. et de l'E.H.E.S.S.

4. Les résultats de ces deux contrats ont été consignés dans deux rapports remis en septembre 1982 aux organismes financeurs. Une partie en a été publiée sous la forme d'un numéro spécial de la revue du CILAC, L'Archéologie industrielle en France, $\mathrm{n}^{\circ} 11$, juin 1985, $164 \mathrm{p}$.

5. Le rapport de synthèse (148 p.) remis en avril 1985 au C.N.R.S., qui a donné lieu à une diffusion restreinte en multigraphie, doit paraitre en 1990 dans une version enrichie et actualisée, parallèlement à la publication d'une série de contributions spécifiques de plusieurs chercheurs dans un numéro spécial des Cahiers d'Histoire et de Philosophie des Sciences, organe de la Société française d'Histoire des Sciences et des Techniques. Certains aspects de cette recherche ont, d'autre part, été présentés lors de deux manifestations dont les actes ont été publiés : le VIIe Symposium international de l'ICOTHEC (Cologne, septembre 1984), et la Table Ronde sur les brevets organisée par le C.N.R.S. (I.H.M.C.) et l'Université de Paris-IV (Centre de Recherche en Histoire de l'Innovation) à Gif-sur-Yvette les 3 et 4 décembre de la même année.

6. Rapport final remis au C.N.R.S. en 1987.

7. L'équipe envisage actuellement de collaborer avec le C.E.R.A.R.E. de Mulhouse (Centre Rhénan d'Archives et de Recherches Économiques) pour l'exploitation d'une partie du fonds de la S.A.C.M. (Société Alsacienne de Constructions Mécaniques).

\section{AUTEURS}

\section{SERGE BENOIT}

Serge BENOIT est chargé de recherche au C.N.R.S. et chargé de conférences à l'E.H.E.S.S. 
GÉRARD EMPTOZ

Gérard EMPTOZ est maître de conférences de Chimie à l'I.U.T d'Orsay et chargé de conférences à l'E.H.E.S.S

\section{CLAUDINE FONTANON}

Claudine FONTANON est chef de travaux à l'E.H.E.S.S., collaboratrice du C.D.H.T. 